\title{
Automated Pattern-Based Pointcut Generation
}

\author{
Mathieu Braem, Kris Gybels, Andy Kellens` Wim Vanderperren \\ Vrije Universiteit Brussel, Pleinlaan 2, 1050 Brussels, Belgium \\ $\{$ mbraem,kgybels,akellens,wvdperre $\} @$ vub.ac.be
}

\begin{abstract}
One of the main problems in Aspect-Oriented Software Development is the so-called fragile pointcut problem. Uncovering and specifying a good robust pointcut is not an easy task. In this paper we propose to use Inductive Logic Programming, and more specifically the FOIL algorithm, to automatically identify intensional (or "pattern-based") pointcuts. We present the tool chain we implemented to induce a pointcut given a set of identified joinpoints. Using several realistic medium-scale experiments, we show that our approach is able to automatically induce robust pointcuts for a set of joinpoints.
\end{abstract}

\section{Introduction}

Separation of concerns [29] is a crucial property for realizing comprehensible and maintainable software. Current software engineering paradigms do however not always succeed in cleanly modularizing all concerns. Consequently, these concerns are spread and repeated over several modules in the system. Due to this code duplication, it becomes very hard to alter such concerns within the system. These concerns are called crosscutting because the concern virtually crosscuts the decomposition of the system. Typical examples of crosscutting concerns are debugging concerns such as logging [19] and contract verification [33], security concerns [8] such as confidentiality and access control, and business rules [28,9] that describe business-specific logic.

Aspect-Oriented Software Development aims to provide a solution for these crosscutting concerns [19]. To this end, AOSD introduces an additional module construct, named an aspect. Traditional aspects consist of two main parts: a pointcut definition and an advice. Points in the program's execution where an aspect can be applied are called joinpoints. The declarative pointcut language allows to concisely describe a set of joinpoints where the aspect should be applied. The advice is the concrete behavior that is to be executed at certain joinpoints, typically before, after or around the original behavior of the joinpoints.

Since existing software systems can benefit from the advantages of AOSD as well, a number of techniques have been proposed to identify crosscutting concerns in existing source code (aspect mining) and transform these concerns into aspects (aspect refactoring). When refactoring a concern to an aspect, a

* Ph.D. scholarship funded by the "Institute for the Promotion of Innovation through Science and Technology in Flanders" (IWT Vlaanderen). 
pointcut must be written for this aspect. Pointcut languages like for instance the CARMA pointcut language allow specifying intensional (or "pattern-based") pointcuts, so that the pointcut does not easily break when the base code is changed [11]. While existing aspect refactoring techniques also automatically generate a pointcut, they typically only provide an enumerative pointcut, which is fragile with respect to evolution of the base program. Turning this pointcut into a pattern-based pointcut is left to be done manually by the developer.

In this paper we propose to exploit Inductive Logic Programming techniques to automatically deduce an intensional pointcut from a given set of joinpoints. The next section details the problem of uncovering intensional pointcuts and introduces the running example used throughout this paper. Section 3 introduces Inductive Logic Programming and the concrete algorithms used and in section 4 we apply ILP for automatically generating intensional pointcuts. Afterwards, we present the tools created to support our approach, compare with related work and state our conclusions.

\section{Background and Problem Statement}

\subsection{Pattern-Based Pointcuts}

The main problem in maintaining aspect-oriented code is the so-called fragile pointcut problem [21]. Pointcuts are deemed fragile when seemingly innocent changes to the base program, such as renaming or relocating a method, break a pointcut such that it no longer captures the joinpoints it is intended to capture. When code is added to a program and introduces new joinpoints in the joinpoint model of the program, pointcuts are similarly considered fragile when some of these new joinpoints should be captured by the pointcut but it fails to do so.

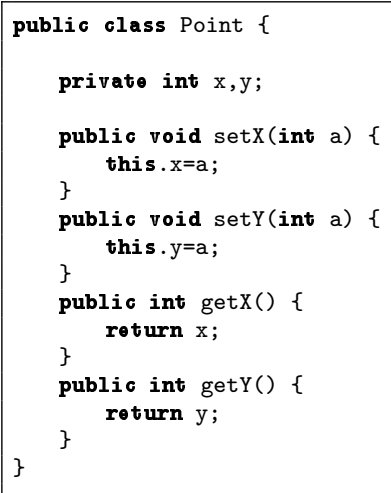

Fig. 1. A simple Point class 
As described in our previous work [11] and that of others [20], pointcuts are particularly fragile when they are written in an enumerative style. As an example take the Point class of figure 1. When adding an observer aspect, we need a pointcut that captures all executions of methods on the Point class that are state changing. A purely enumerative pointcut is shown in figure 2 .

The pointcut language used in the figure and the remainder of the paper is based on CARMA but is restricted to a static joinpoint model, we'll refer to it as $\mathrm{PAGH}^{1}$ to make the distinction. CARMA [11] uses a fully dynamic joinpoint model, which for example allows conditions in pointcuts on the values associated with joinpoints. PAGH has a purely static joinpoint model, which effectively equates joinpoints with shadow joinpoints. An extension of the work presented here that takes a dynamic joinpoint model into account is left for future work. The important point however is that PAGH retains other features of CARMA which allow writing advanced intensional pointcuts: the use of logic variables, recursion and full access to the static shadow joinpoint model of the program.

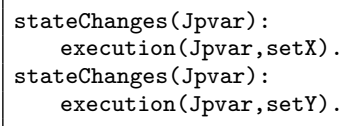

Fig. 2. A pointcut for the Observer aspect, written in a purely enumerative style.

The pointcut of figure 2 matches if the joinpoint at hand is either the execution of method setX or the execution of method setY. Such an enumeratively described pointcut obviously breaks easily. For example, when we evolve the point class to a three-dimensional point and add a setZ method, the stateChanges pointcut does not match the added method and thus fails to comply with the intention of capturing all methods that change the state of a Point object.

The problem with enumerative pointcuts is of course the motivation for writing pointcuts in a more pattern-based style, exploiting a pattern that is exhibited by the joinpoints that should be captured. The pointcut in figure 3 uses quantification over the names of methods that start with set. It remains consistent when evolving the point to a three-dimensional point. However, consider for example the addition of a reset method that resets the $\mathrm{x}$ and $\mathrm{y}$ dimension of the point to the default values. This method does not have the begins with the keyword set pattern in common with the other state changing methods. Conversely, consider the addition of a method setting which simply returns the value of a setting, rather than doing any assignments. This method also exhibits the begins with keyword set pattern but should in fact not be captured by the pointcut. We can capture the reset and setting methods as a deviation from the pattern by in-

\footnotetext{
${ }^{1}$ The name refers to a concept from the same realm as does Karma, see http://memory-alpha.org/en/wiki/Pagh
} 
cluding an extra condition that the name of the method may also be reset and should not be setting, but this tends to add an enumerative list of exceptions to the pointcut.

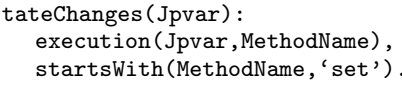

Fig. 3. A pointcut for the Observer aspect, written in a pattern-based style.

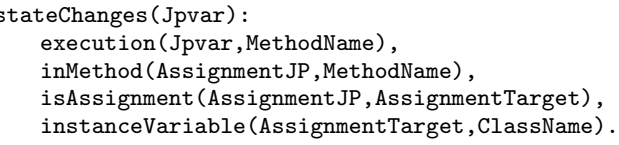

Fig. 4. A pointcut for the observer

Using an advanced pointcut language that gives access to the full static joinpoint model of methods, it is possible to exploit a more robust pattern [11]. Figure 4 illustrates a pointcut that exploits the pattern that all the state changing methods contain an assignment to an instance variable of an object. This pointcut does not break when adding the setting or reset methods.

\subsection{Automated Support for Pattern-Based Pointcuts}

The area of aspect refactoring and aspect mining is a particularly interesting research area within AOSD that is currently being explored. In performing aspect mining and refactoring, the problem crops up of finding a pointcut for the newly created aspect. Also, as with object-oriented refactoring, research is being performed on how to automate these refactorings using tool support. In such tools, it would be interesting to be able to automate the step of generating a pattern-based pointcut as well. Currently, most proposals for automating aspect refactoring simply generate an enumerative pointcut, which then too easily breaks when the program is evolved after refactoring.

In this paper we present the results of using a specific machine learning technique for deriving a pattern exhibited by examples. In particular we use Inductive Logic Programming, which is in fact an algorithm that works similarly to the process we've described in the previous section for coming to an evolutionrobust pattern-based pointcut. We further describe this relation informally in the next section, and present in detail the ILP algorithm. 


\section{Inductive Logic Programming}

\subsection{Logic Induction of Pointcuts}

The algorithm of logic induction is similar to the process we followed in section 2.1 for coming to a more evolution-robust pattern-based pointcut. Informally, the way ILP works and the relationship to this manual process is as follows:

positive examples: ILP takes as input a number of positive examples, in our setting of deriving pattern-based pointcuts these would be joinpoints that the pointcut should capture.

background information: A second input to ILP is background information on the examples. In our setting, these would be the result of predicates in the pointcut language that are true for the joinpoints, or in other words, the data associated with the joinpoints. Such as the name of the message of the joinpoint, the type of the joinpoint (message, assignment, ...), in which method or class the joinpoint occurs.

induction: ILP follows an iterative process of inducing a logic rule for combinations of the positive examples. This is similar to the manual process we followed in the previous section: we take two examples such as the methods setX and setY, and find that in the background information the fact that the names of the methods start with set holds true.

negative examples: ILP also takes as input a number of negative examples, the rules that are derived during the iterative induction should never cover negative examples. Negative examples effectively force the algorithm to use other information of the background in the induced rules. This is similar to the process followed in the previous section where we added a setting method which should not be covered by the pointcut.

\section{$3.2 \quad$ FOIL}

In this paper we use the FOIL ILP algorithm [30]. FOIL learns hypotheses which are sets of first-order rules, similar to Horn clauses. However, since no literals containing function symbols are allowed, the rules are more restricted than Horn clauses. On the other hand, the rules are more expressive because literals appearing in the body of the rules may be negated.

Pseudo-code for the algorithm is shown in figure 5. The algorithm takes a top-down approach to ILP. Starting with the most general rule, FOIL specializes it until no more negative examples are covered. The algorithm involves a double loop to find suitable queries. In the outer loop the algorithm generates rules, each time starting with the most general rule, covering all examples. In the inner loop, it adds clauses to the rule, until no more negative examples are covered. The algorithm halts when all positive examples have been covered.

The algorithm generates candidate literals based on the literals and variables already present in the rule, and on predicates found in the background information. Suppose the current rule is Predicate $\left(X_{1}, X_{2}, \ldots, X_{k}\right) \leftarrow$ Literal $_{1} \ldots$ Literal $_{n}$. FOIL now considers the following literals for addition as Literal $_{n+1}$. 


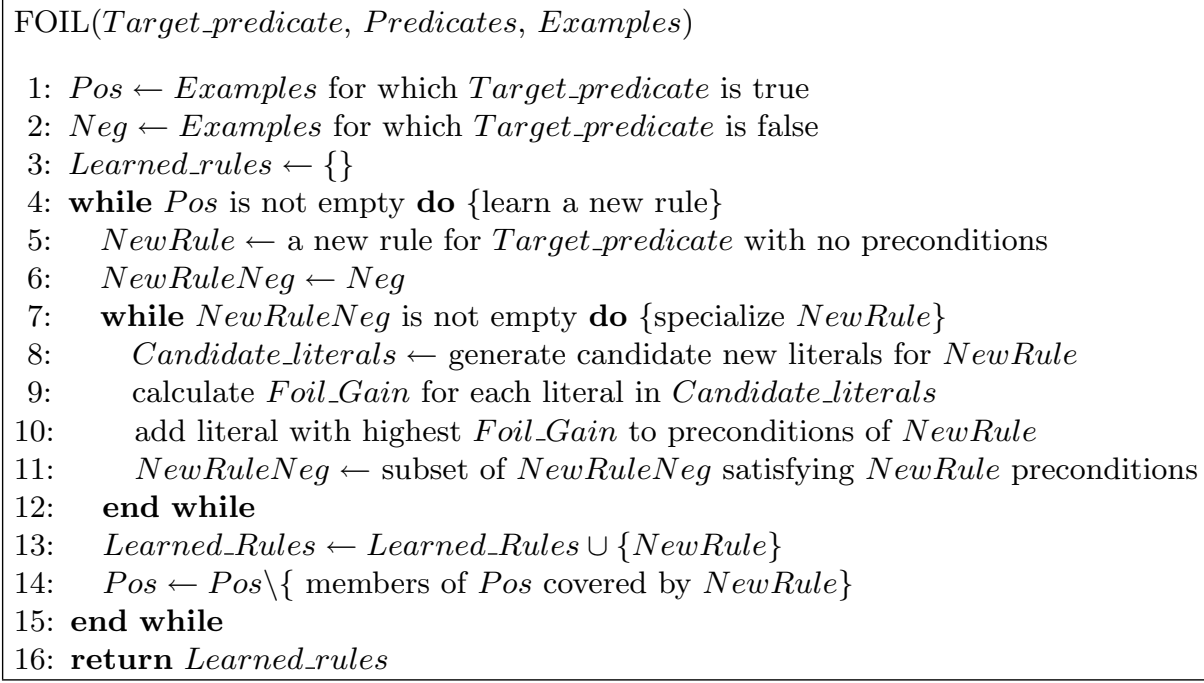

Fig. 5. FOIL Algorithm

- $Q\left(V_{1}, \ldots, V_{r}\right)$, where $Q$ is predicate occurring in the background information and where $V_{i}(\forall i, 0<i<r)$ is either a new variable or a variable already present in the rule. At least one of the variables $V_{i}$ has to be present in rule.

- Equal $\left(X_{j}, X_{k}\right)$, where $X_{j}$ and $X_{k}$ are variables already present in the rule.

- The negation of the literals formed in the rules above.

At each step of the inner loop a heuristic function is evaluated for all candidate literals. The result of this function shows how much the rule gains from adding this literal. The candidate literal which results in the highest gain is chosen as the next literal. This gain function, shown in figure 6 , is a simple measure, based on the comparison of the number of covered positive $(p)$ and negative $(n)$ examples before $\left(p_{0}, n_{0}\right)$ and after $\left(p_{1}, n_{1}\right)$ the literal is added to the rule. The numbers of bindings that remain positive $(t)$ after adding the literal to the rule is factored in.

$$
\text { Foil_Gain }(L, R)=t\left(\log _{2} \frac{p_{1}}{p_{1}+n_{1}}-\log _{2} \frac{p_{0}}{p_{0}+n_{0}}\right)
$$

Fig. 6. Foil_Gain function 


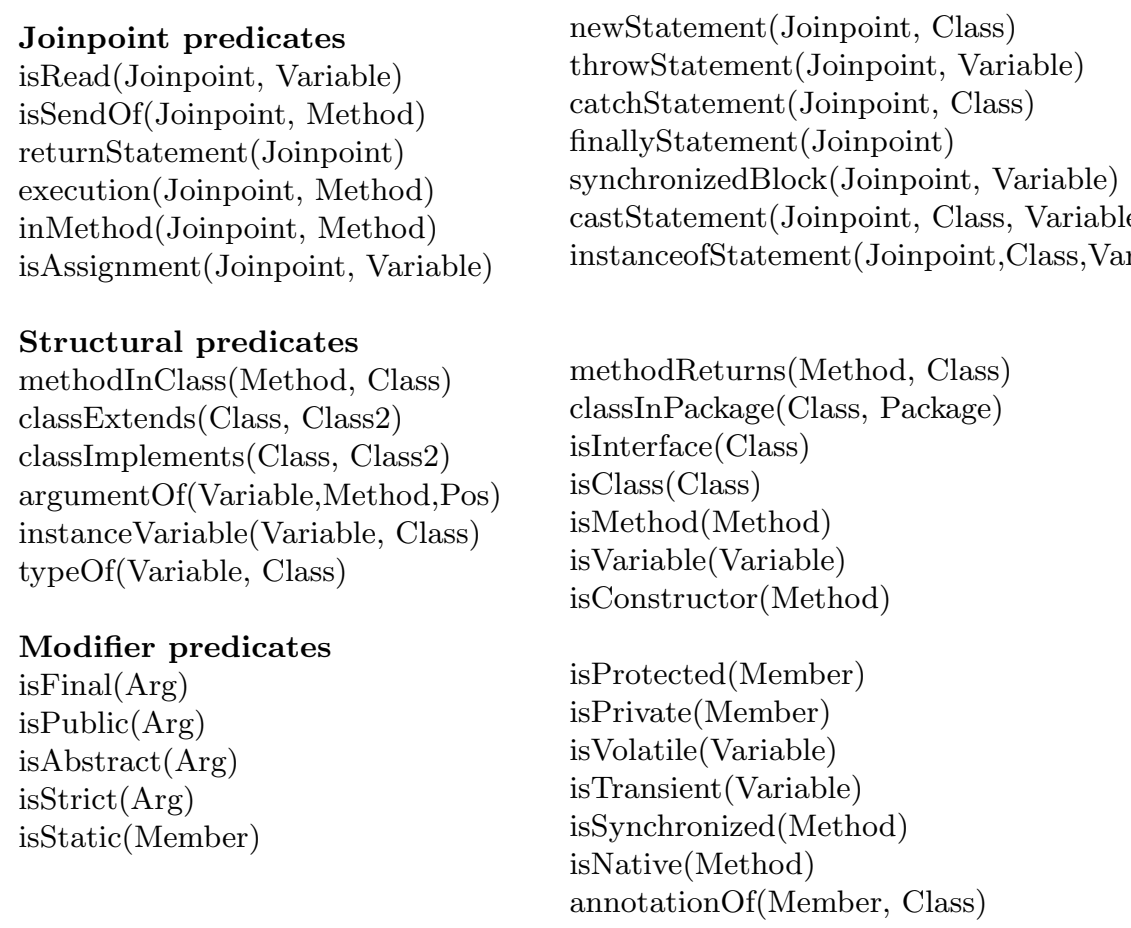

Fig. 7. Predicates available in the PAGH crosscut language to select joinpoints, the solutions for these predicates are used as background information for the ILP algorithm.

\section{Applying ILP for Pointcut Abstraction}

The FOIL algorithm is able to find rules from a set of logic facts. It requires a number of positive examples and a set of negative examples to avoid oversimplification. In addition, it expects a sufficiently large set of background information in order to be able to induce a rule. The positive examples for FOIL are the joinpoints where the aspect needs to be applied. They can either be manually selected or automatically using for example an aspect mining technique. All other joinpoints are defined as negative examples for the ILP algorithm. As background information, we construct a logic database consisting of the information that is normally available in the pointcut language on these joinpoints. These are the solutions of the predicates shown in figure 7 , which also includes predicates about the relationships between classes etc. Because this pointcut language is based on a purely static joinpoint model, these solutions can be determined using only the program's source or compiled representation, i.e. compiled Java classes.

The algorithm will induce a pointcut that captures exactly the joinpoints currently in the program that should be captured (the positive examples), and 
besides jp0 and jp5 are marked as negative examples. We give the FOIL algorithm the positive examples stateChanges (jp0) and stateChanges ( jp5). The resulting rule is shown in figure 9 . The pointcut selects all executions of methods that contain an assignment.

The resulting pointcut is clearly not very robust. An evolution that easily breaks the pointcut would be to have a getX method that does an assignment to a local variable which does not mean that that method changes the state of an object, yet its execution would be captured by the pointcut. This result is however not very surprising: the Point class is small and does not include nonstate changing methods that do assignments to local variables which would have served as a negative example for the FOIL algorithm. As the induced pointcut covers all positive examples and no negative ones, the induction stops and no further predicates from the background information are used to limit the rule to only the positive examples. The ILP algorithm works better on larger programs, so that more negative examples are available to avoid oversimplified patternbased pointcuts.

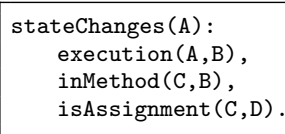

Fig. 9. Induced stateChanges pointcut.

In order to have a more realistic example, we apply our experiment to the Point class bundled with Java. We do not include a full listing of the generated background, but instead we give some statistics about the generated facts. Table 1 compares the number of facts found in the AWT Point class to the number of facts from the basic Point example.

Table 1. Generated facts statistics

\begin{tabular}{|l|c|c|c|}
\hline & \# Classes & \# Facts & \# Joinpoints \\
\hline Toy example & 1 & 71 & 10 \\
AWT Point class & 1 & 364 & 70 \\
Complete AWT library & 362 & 276863 & 65060 \\
\hline
\end{tabular}

We identify four execution joinpoints in the AWT Point class where a state changing method is invoked and input them as positive examples to the algorithm. The remaining 66 joinpoints are defined as negative examples. The resulting pointcut is shown in figure 10 . In this case, the algorithm generates a pointcut that is sufficiently robust for evolution: it is in fact the same pointcut we determined manually in section 2.1. 


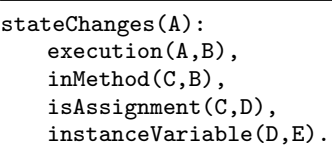

Fig. 10. Resulting pointcut when applying our approach to the AWT Point class.

\subsection{Extended experiments}

In order to provide a limited evaluation of our approach, we conduct several more involved experiments using the state-changes example on the Java AWT framework.

Large fact database: We apply our approach to the complete Java AWT library in order to evaluate whether our approach still returns a useful result when the number of facts is very large. This library contains approximately 362 classes and generates more than 250000 facts. The result is the same as for the Java AWT Point class alone: the same pointcut as was determined manually in section 2.1 is induced. For a performance evaluation, we refer to section 5 .

Negation: One of the distinguishing features of the FOIL algorithm in comparison to other ILP algorithms is its ability to induce rules containing negations. As a variation of the state changing methods example, we need a pointcut for the executions of methods that change the observable representation of an object. This means the method does assignments to instance variables that are not declared transient using the modifier transient in Java: conceptually, these fields are not part of the object's persistent state and are not retained in the object's serialization. This is used for example when a class defines a cache in order to optimize some parts of its operations. As such, observers do not need to be notified when transient fields are altered. When applying this experiment to the Java AWT library, our algorithm induces the rule shown in figure 11, which in comparison to the pointcuts induced above adds exactly the properties in the background to distinguish these joinpoints from the negative examples that we would expect it to add, i.e. the fact that the instance variables being assigned to are not declared transient.

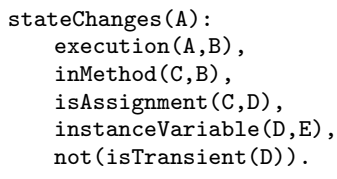

Fig. 11. Resulting pointcut for non-transient field assignments in Java AWT. 
InEquality: The FOIL algorithm is also able to induce inequality for certain rule variables. For example, suppose we want to detect all methods that contain "illegal" assignments, namely assignments to instance variables of other classes. The rule of figure 12 is induced when we apply this experiment to the AWT library. This rule declares that a method is illegally state changing when it contains an assignment to an instance variable that does not belong to the same class as the method.

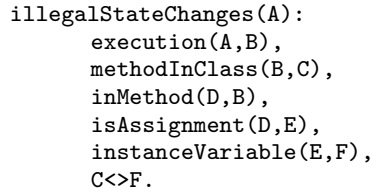

Fig. 12. Resulting pointcut for field assignments from a different class than the class defining the field.

Recursion: Another advantage of the FOIL algorithm is its ability to induce recursive rules. For example, suppose we redefine state changing methods to also include execution joinpoints of methods that indirectly change the state of an object by invoking a method that is state changing. This is useful for implementations of the observer aspect that take into account the jumping aspect problem $[4,11]$. In order to capture this pattern robustly, two pointcut rules are required, one of which is recursive. In this experiment our ILP implementation however did not induce such a recursive pointcut rule although theoretically the algorithm is able to induce recursive rules. The algorithm induces several rules that are unnecessarily complicated, depending on information that is irrelevant to the state changing concern. This pointcut breaks easily when the base program evolves because it is concerned with too much information. However, when we use method names as positive examples rather than joinpoints, a recursive rule is induced which does not exhibit such fragility issues, the resulting rule is shown in figure 13. All that would be necessary to turn this into a pointcut is an extra condition which gets the joinpoint associated with the method name. A possible reason that the algorithm doesn't try adding this condition may have to do with the gain function, but this needs to be investigated further in future work.

\section{Tool Support}

Our approach is supported by a fully automatic tool chain, which is illustrated in figure 14. The tool chain consists of the following tools:

- FactGen: This tool translates a range of Java class files and/or jar files to a set of facts representing these classes. The tool uses the javassist library [7] 


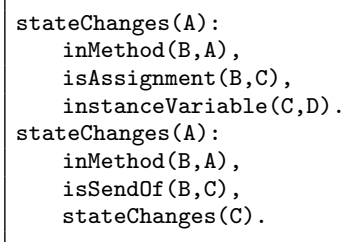

Fig. 13. Recursive stateChanges rule.

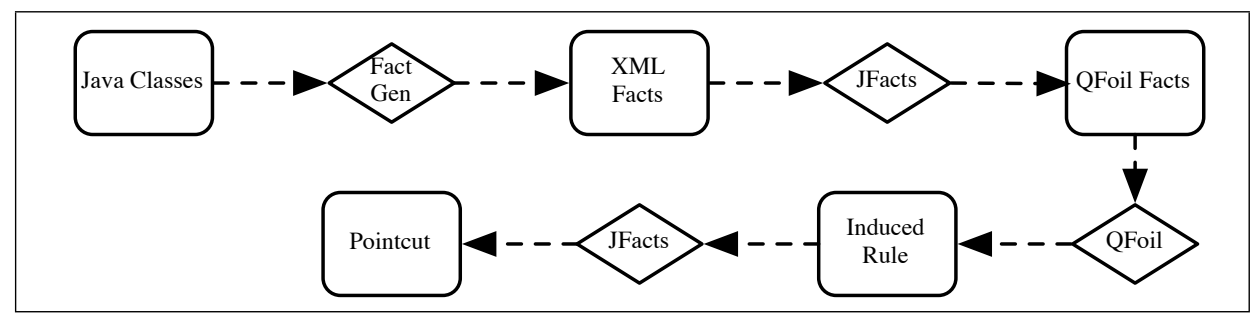

Fig. 14. The tool chain for inducing pointcuts in a logic pointcut language over static joinpoints in Java code.

to process the binary class files. The javassist library provides a high-level reflective API that allows to inspect the full Java byte code, including method bodies. The output of the FactGen tool is the fact representation in XML format.

- JFacts: This tool allows to translate logic predicates from one syntax into another. Currently, the tool supports the FactGen's XML syntax, QFoil's syntax, CARMA's syntax and PAGH and Prolog syntax.

- QFoil: This tool is the implementation of the FOIL ILP algorithm by Ross Quinlan [31]. It takes a set of facts and a set of positive examples as input (negative examples are implicitly assumed) and tries to induce a logic rule that covers all of the positive examples and rejects all of the negative examples. This implementation of FOIL is particularly interesting because of its performance (see the benchmarks in the next paragraph).

In order to evaluate our approach performance-wise, we conduct several benchmark experiments with an increasingly large number of facts. The experiments were done using the state changing methods example. Table 2 shows the results $^{2}$. In all cases, except for the toy Point class of course, the rule from Figure 10 was induced. The performance results are acceptable as the time required is not much more than compiling such a large set of classes. Considering the premature stage of the FactGen and JFacts tools, we believe that a significant improvement is still possible there.

${ }^{2}$ The timings were performed on an Intel Pentium 4 3Ghz. Each timing represents the average time of a single experiment, based on 100 experiments. 
Table 2. Benchmark results of our prototype tool chain.

\begin{tabular}{|l|ccccc|}
\hline & \# classes & \# facts & \# joinpoints & FactGen+JFacts (s) & QFOIL (s) \\
\hline Toy Point class & 1 & 71 & 10 & 0.461 & 0.01 \\
AWT Point class & 1 & 364 & 70 & 0.5902 & 0.0142 \\
25 classes from AWT & 25 & 11622 & 2855 & 1.8098 & 0.8779 \\
50 classes from AWT & 50 & 42870 & 10982 & 3.9702 & 5.4671 \\
75 classes from AWT & 75 & 79403 & 21367 & 6.5163 & 4.4448 \\
100 classes from AWT & 100 & 88236 & 23409 & 7.1599 & 5.4526 \\
AWT (no subpackages) & 118 & 103752 & 27862 & 7.9929 & 7.1708 \\
\hline
\end{tabular}

\section{Related Work}

To our knowledge, there exist few approaches which try to automatically generate pattern-based pointcuts. In previous work [12] we already report on a first attempt for using Inductive Logic Programming in order to derive pattern-based pointcuts. In this work we employ Relative Least General Generalisation [27], an alternative ILP algorithm, instead of the FOIL algorithm. Using RLGG, we are able to derive correct pointcuts for some specific crosscutting concerns in a Smalltalk image. However, due to the limitations of both our implementation as well as the applied ILP algorithm (for instance, the algorithm does not support negated literals), our RLGG-based technique often results in pointcuts that suffer from some fragility: the resulting pointcuts for example frequently contain redundant literals referring to the names of specific methods or classes, which of course easily breaks the pointcut when these names are changed. Furthermore, our earlier work suffers from serious scalability issues. In the context of Adaptive Programming [24], an approach has been developed for automatically deducing traversal strategies, which are AP's counterpart of pointcuts [25], from a given class and object graph.

As mentioned earlier, the major area of application of our technique lies in the automated refactoring of crosscutting concerns in pre-AOP code into aspects. Quite a number of techniques exist $[13,26,22,15]$ which propose refactorings in order to turn object-oriented applications into aspect-oriented ones. However, these techniques do not consider the generation of pattern-based pointcuts. Instead they propose to automatically generate an enumeration-based pointcut which, optionally, can be manually turned into a pattern-based pointcut by the developer. As is pointed out by Binkley et al. [2], our technique is complementary with these approaches as it can be used to both improve the level of automation of the refactoring, as well as the evolvability of the refactored aspects.

In the context of aspect mining, which is closely related to object-to-aspect refactorings, a wealth of approaches are available that allow for the identification of crosscutting concerns in an existing code base. The result of such a technique is typically an enumeration of joinpoints where the concern is located. Ceccato et al. [6] provide a comparison of three different aspect mining techniques: identifier analysis, fan-in analysis and analysis of execution traces. Breu and Krinke propose an approach based on analyzing event traces for concern identification [3]. 
Bruntink et al. [5] make use of clone detection techniques in order to isolate idiomatically implemented crosscutting concerns. Furthermore, several tools exist that support aspect mining activities by allowing developers to manually explore crosscutting concerns in source code, such as the aspect mining tool [14], FEAT [32], JQuery [17] and the Concern Manipulation Environment [16]. These approaches are complementary with our approach in that the joinpoints they identify can serve as positive examples for our ILP algorithm.

\section{Conclusions and Future Work}

In this paper we present our approach using Inductive Logic Programming for generating a concise and robust pointcut from a given enumeration of joinpoints. We report on several successful experiments that apply our approach to a realistic and medium-scale case study. We have applied our approach to a CARMA-based logic pointcut language restricted to a static joinpoint model, dubbed PAGH. In future work we will consider tackling full CARMA which requires taking into account in the background information that joinpoints and joinpoint shadows are not equated as in PAGH. Our approach can easily be applied to for example AspectJ [18] as well by translating PAGH pointcuts to AspectJ pointcuts. However, the FOIL algorithm must then be restricted to not generate pointcuts using features of PAGH that can not be translated to AspectJ: variables can only be used once in a pointcut (except when using the "if" restrictor in AspectJ), recursive named pointcuts are not possible, and only some uses of the structural predicates can be translated. Other points left for future work are:

- Multiple Results: Our current tools only generate one pointcut for a given set of joinpoints. In some cases, most notably when there is few background information (i.e. a small number of little classes), several alternative pointcuts are possible. Our current approach has a bias for short, non-negative and non-recursive rules. As we have described in the paper, this might not always lead to a (good) result. Therefore, it would be useful to allow presenting multiple pointcut results. An interesting research topic in this context would consist of uncovering poincut patterns and anti-patterns that might be used to guide the selection and generation process.

- Other Algorithms: There exist several algorithms for Inductive Logic Programming. In previous work, we conduct several small-scale experiments with the Relative Least General Generalization (RLGG) [27] algorithm in an aspect mining context [12]. Having several algorithms might improve the quality of the selected results to the end-user. For example, solutions that are induced by more than one algorithm might be better.

- Run-Time Information: Our current approach only analyzes the static program information to induce pointcuts. Pointcuts that require run-time program information, such as stateful aspects [10], cannot be induced. For this end, facts representing the run-time behavior of the program are necessary. We are currently investigating whether it is possible to induce such dynamic pointcuts using several program traces as background information. 
- Tool Integration: Although our current tool works fully automatically, it is a stand-alone command-line tool that is not integrated in an IDE. We plan to develop an Eclipse plugin for our tool. This plugin can then be a basis for inducing pattern-based pointcuts by other plugins which provide support for the refactoring process.

\section{References}

1. Mehmet Akşit, editor. Proc. 2nd Int' Conf. on Aspect-Oriented Software Development (AOSD-2003). ACM Press, March 2003.

2. D. Binkley, M. Ceccato, M. Harman, F. Ricca, and P. Tonella. Automated refactoring of object oriented code into aspects. In 21st IEEE International Conference on Software Maintenance (ICSM), 2005.

3. Silvia Breu and Jens Krinke. Aspect mining using event traces. In 19th International Conference on Automated Software Engineering, pages 310-315, Los Alamitos, California, September 2004. IEEE Computer Society.

4. Johan Brichau, Wolfgang De Meuter, and Kris De Volder. Jumping aspects. In C. Lopes, L. Bergmans, M. D'Hondt, and P. Tarr, editors, Workshop on Aspects and Dimensions of Concerns (ECOOP 2000), June 2000.

5. M. Bruntink, A. van Deursen, R. van Engelen, and T. Tourwé. An evaluation of clone detection techniques for identifying crosscutting concerns. In Proceedings of the IEEE International Conference on Software Maintenance (ICSM). IEEE Computer Society Press, 2004.

6. M. Ceccato, M. Marin, K. Mens, L. Moonen, P. Tonello, and T. Tourwé. A qualitative comparison of three aspect mining techniques. In Proceedings of the 13th International Workshop on Program Comprehension (IWPC 2005), pages 13-22. IEEE Computer Society Press, 2005.

7. Shigeru Chiba and Muga Nishizawa. An easy-to-use toolkit for efficient Java bytecode translators. In GPCE '03: Proceedings of the second international conference on Generative programming and component engineering, pages 364-376, New York, NY, USA, 2003. Springer-Verlag New York, Inc.

8. Bart De Win, Wouter Joosen, and Frank Piessens. Developing secure applications through aspect-oriented programming. pages 633-650. Addison-Wesley, Boston, 2005.

9. Maja D'Hondt and Viviane Jonckers. Hybrid aspects for weaving object-oriented functionality and rule-based knowledge. In Lieberherr [23], pages 132-140.

10. Rémi Douence, Pascal Fradet, and Mario Südholt. Composition, reuse and interaction analysis of stateful aspects. In Lieberherr [23], pages 141-150.

11. Kris Gybels and Johan Brichau. Arranging language features for pattern-based crosscuts. In Akşit [1], pages 60-69.

12. Kris Gybels and Andy Kellens. An experiment in using inductive logic programming to uncover pointcuts. In First European Interactive Workshop on Aspects in Software, September 2004.

13. Stefan Hanenberg, Christian Oberschulte, and Rainer Unland. Refactoring of aspect-oriented software. In 4th Annual International Conference on ObjectOriented and Internet-based Technologies, Concepts, and Applications for a Networked World, 2003.

14. J. Hannemann. The Aspect Mining Tool web site. http://www.cs.ubc.ca/labs/spl/ projects/amt.html. 
15. Jan Hannemann, Gail Murphy, and Gregor Kiczales. Role-based refactoring of crosscutting concerns. In Peri Tarr, editor, Proc. 4rd Int' Conf. on Aspect-Oriented Software Development (AOSD-2005), pages 135-146. ACM Press, March 2005.

16. William Harrison, Harold Ossher, Stanley M. Sutton Jr., and Peri Tarr. Concern modeling in the concern manipulation environment. IBM Research Report RC23344, IBM Thomas J. Watson Research Center, Yorktown Heights, NY, September 2004.

17. Doug Janzen and Kris De Volder. Navigating and querying code without getting lost. In Akşit [1], pages 178-187.

18. G. Kiczales, E. Hilsdale, J. Hugunin, M. Kersten, J. Palm, and W. G. Griswold. An overview of AspectJ. In J. L. Knudsen, editor, Proc. ECOOP 2001, LNCS 2072, pages 327-353, Berlin, June 2001. Springer-Verlag.

19. Gregor Kiczales, John Lamping, Anurag Mendhekar, Chris Maeda, Cristina Lopes, Jean-Marc Loingtier, and John Irwin. Aspect-oriented programming. In Mehmet Akşit and Satoshi Matsuoka, editors, 11th Europeen Conf. Object-Oriented Programming, volume 1241 of LNCS, pages 220-242. Springer Verlag, 1997.

20. Gregor Kiczales and Mira Mezini. Separation of concerns with procedures, annotations, advice and pointcuts. In European Conference on Object-Oriented Programming, ECOOP 2005, 2005.

21. Christian Koppen and Maximilian Störzer. PCDiff: Attacking the fragile pointcut problem. In Kris Gybels, Stefan Hanenberg, Stephan Herrmann, and Jan Wloka, editors, European Interactive Workshop on Aspects in Software (EIWAS), September 2004 .

22. Ramnivas Laddad. Aspect-oriented refactoring, dec 2003.

23. Karl Lieberherr, editor. Proc. 3rd Int' Conf. on Aspect-Oriented Software Development (AOSD-2004). ACM Press, March 2004.

24. Karl J. Lieberherr. Adaptive Object-Oriented Software: the Demeter Method with Propagation Patterns. PWS Publishing Company, Boston, 1996.

25. Karl J. Lieberherr, Jeffrey Palm, and Ravi Sundaram. Expressiveness and complexity of crosscut languages. In Gary T. Leavens, Curtis Clifton, and Ralf Lämmel, editors, Foundations of Aspect-Oriented Languages, March 2005.

26. Miguel Pessoa Monteiro. Catalogue of refactorings for aspectj. Technical Report UM-DI-GECSD-200401, Universidade Do Minho, 2004.

27. S. Muggleton and C. Feng. Efficient induction in logic programs. In S. Muggleton, editor, Inductive Logic Programming, pages 281-298. Academic Press, 1992.

28. H. Ossher and P. Tarr. The shape of things to come: Using multi-dimensional separation of concerns with Hyper/J to (re)shape evolving software. Comm. ACM, 44(10):43-50, October 2001.

29. D. L. Parnas. On the criteria to be used in decomposing systems into modules. Comm. ACM, 15(12):1053-1058, December 1972.

30. J. Ross Quinlan. Learning logical definitions from relations. Machine Learning, 5(3):239-266, August 1990.

31. Ross Quinlan. Qfoil: the reference foil implementation. Home page at http: //www.rulequest.com/Personal/, 2005.

32. Martin P. Robillard and Gail C. Murphy. Automatically inferring concern code from program investigation activities. In Proceedings of Automated Software Engineering (ASE) 2003, pages 225-235. IEEE Computer Society, 2003.

33. Wim Vanderperren, Davy Suvée, and Viviane Jonckers. Combining AOSD and CBSD in PacoSuite through invasive composition adapters and JAsCo. In Net.ObjectDays 2003, pages 36-50, September 2003. 